\title{
We've got mice...
}

\section{A new zebrafish microchip - but this week it has to be mainly mice.}

\author{
Mouse Universal Reference Total RNA \\ Clontech \\ www.clontech.com \\ Raising the standards
}

Clontech claims that comparisons of your microarray data just got easier with the introduction of Mouse Universal Reference Total RNA. This universal reference RNA is made by pooling the total RNA extracts from a collection of different tissues, yielding a mixture with the broadest possible gene representation available. Production is on an industrial scale, which minimizes variation between lots. Each kit is supplied with sufficient RNA for up to 80 microarray experiments, facilitating comparison of data sets from different microarray experiments. It can be used with any standard array or labelling method.

\section{IHC kit for rodent tissues}

\section{InnoGenex}

www.innogenex.com

Close encounters

This kit is designed for immunohistochemical (IHC) staining of tissues from species closely related to the primary antibody-producing species. The biotinylated secondary antibodies have been treated with solidphase adsorption against protein extracts from mouse, rat or rabbit tissues to eliminate cross-reactivities and produce clean results with low background. Serial amplifications allow high sensitivity, retaining the high specificity inherent in the antibody-antigen and biotin-streptavidin interactions. The kits are available for mouse antibodies on rat tissues, rabbit antibodies on mouse or rat tissues, rat antibodies on mouse tissues and mouse antibodies on rabbit tissues, with a choice of AEC, BCIP/NBT, DAB, Fast Red, Phycoerythrin, FluorBlue, FluorGreen or FluorRed chromogen/substrate. Each kit contains sufficient reagents for staining 60 slides. Results are obtained in about two hours.

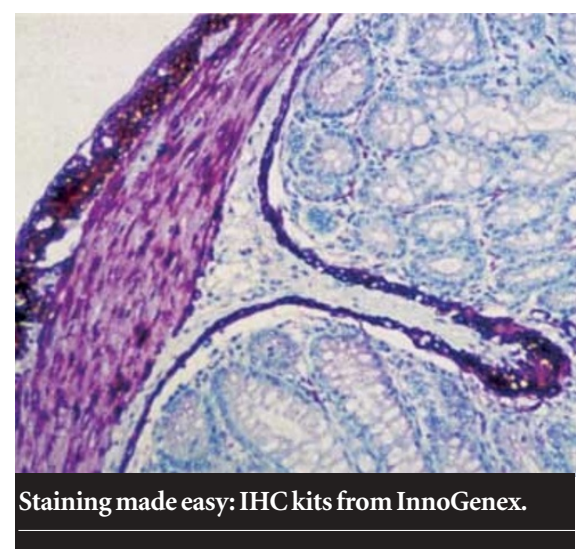

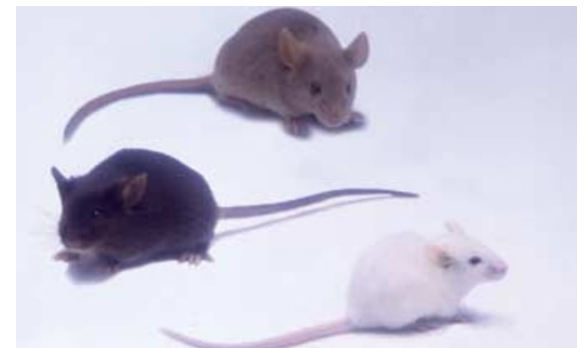

Focus on tau proteins with Taconic.

Mouse model for neurological disorders

Taconic www.taconic.com

It takes tau to tangle

Taconic's tau micro-injected mouse model carries the transgene for the human P301L mutation of the microtubule-associated protein tau (MAPT) gene. Fronto-temporal dementia and parkinsonism linked to chromosome 17 have been correlated with mutations in the MAPT gene encoding the tau protein. Motor and behavioural disturbances are observed in approximately $90 \%$ of these mice by 10 months of age, correlating with the development of neurofibrillary tangles. The tau micro-injected mouse is suitable for in vivo research studies of Alzheimer's disease, Pick's disease and other syndromes associated with neurofibrillary tangles.

\section{Zebrafish $14 \mathrm{~K}$ array}

MWG Biotech wWw.THE-MWG.com

\section{For good eggs}

Described as the first commercially available biochip on this important model organism, the zebrafish array contains 14,067 zebrafish genes. Each oligonucleotide represents a protein-coding sequence of the zebrafish genome. The array is designed to be used in embryogenesis research. The zebrafish, as a vertebrate, is especially suitable for this type of research as its eggs are translucent and mature outside the mother's body.

\section{HistoMouse-MAX}

Zymed www.zymed.com

Mouse-to-mouse enquiries

HistoMouse-MAX can be used to detect mouse, rabbit, rat and guinea pig primary antibodies on mouse or rat tissue samples. It contains no biotin or streptavidin, so potential background due to endogenous biotin activity is avoided. Zymed's BEAT (blocking endogenous antibody technology) prevents the secondary antibody from reacting with endogenous Ig. HistoMouse-MAX contains high sensitive peroxidase (HRP) conjugate and uses one step of HRP conjugated to second antibody instead of two steps as in the LAB-SA method. Kits are available in the HRP/DAB system, producing a dark brown stain, and the HRP/AEC system, which creates an intense red.

\section{AnonyMOUSE}

Mouse Specifics www.mousespecifics.com Non-invasive cardiac screening system

AnonyMOUSE is a high-throughput system for recording electrocardiograms (ECGs) in conscious mice without the use of anaesthesia, restraint, surgery or implants. The system detects the electrical signals of the heart through the feet of small rodents, providing heart-rate measurements and showing any evidence of cardiac arrhythmias. AnonyMOUSE has been used successfully to demonstrate ECG properties in dystrophindeficient mice that mirror clinical observations in patients with Duchenne muscular dystrophy.

These notes are compiled in the Nature office from information provided by the manufacturers.

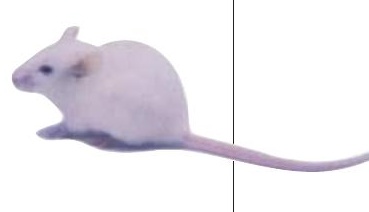

